\title{
AN ELECTRO-MECHANICAL INVESTIGATION OF THE RIEMANN ZETA FUNCTION IN THE CRITICAL STRIP
}

BALTH. VAN DER POL

The theoretical basis underlying the following electromechanical investigations concerns a simple integral representation of the Riemann zeta function in the critical strip. To the right of the critical strip $(\operatorname{Re} s>1)$ we have the usual expression:

$$
\Gamma(s) \cdot \zeta(s)=\int_{0}^{\infty} \frac{1}{e^{x}-1} x^{s-1} d x, \quad \operatorname{Re} s>1
$$

Subtraction from (1) of

$$
\Gamma(s-1) \cdot \alpha^{1-s}=\int_{0}^{\infty} e^{-\alpha x} x^{s-2} d x, \quad \operatorname{Re} s>1, \operatorname{Re} \alpha>0,
$$

yields

(3) $\Gamma(s) \cdot\left\{\zeta(s)-\frac{\alpha^{1-s}}{1-s}\right\}=\int_{0}^{\infty}\left(\frac{1}{e^{x}-1}-\frac{e^{-\alpha x}}{x}\right) x^{s-1} d x, \operatorname{Re} s>0$.

In the integrand of (3) the simple pole at $x=0$ of $\left(e^{x}-1\right)^{-1}$ is compensated by the simple pole with the same residue of $x^{-1} e^{-\alpha x}$. Therefore (3) already converges in the wider band $\operatorname{Re} s>0$. If we now restrict $s$ to the critical strip

$$
0<\operatorname{Re} s<1
$$

we can let $\alpha \rightarrow 0$ in (3), so that we obtain the basic integral

$$
\Gamma(s) \cdot \zeta(s)=\int_{0}^{\infty}\left(\frac{1}{e^{x}-1}-\frac{1}{x}\right) x^{s-1} d x, \quad 0<\operatorname{Re} s<1,
$$

this being the analytical continuation of (1).

The representation (4) of the zeta-function in the critical strip enables us to derive the functional equation of the zeta-function in an extremely simple way. To this end we make use of Legendre's relation ${ }^{1}$

$$
2 \int_{0}^{\infty} \sin x t \frac{1}{e^{2 \pi t}-1} d t=\frac{1}{e^{x}-1}-\frac{1}{x}+\frac{1}{2}=\frac{1}{2} \operatorname{coth} \frac{x}{2}-\frac{1}{x}
$$

Received by the editors March 18, 1947.

${ }^{1}$ See, for example, Whittaker-Watson, Modern analysis, 4th ed., p. 122. 
which is valid for all real values of $x$; furthermore both sides of (5) represent an odd function of $x$. We now restrict $x$ to positive values and subtract

$$
2 \int_{0}^{\infty} \sin x t \cdot \frac{d t}{2 \pi t}=\frac{1}{2}
$$

from (5), so that (5) assumes the self-reciprocal form

$$
2 \int_{0}^{\infty} \sin x t\left(\frac{1}{e^{2 \pi t}-1}-\frac{1}{2 \pi t}\right) d t=\frac{1}{e^{x}-1}-\frac{1}{x}, \quad x>0 .
$$

Substitution of (6) in (4) yields

$$
\Gamma(s) \cdot \zeta(s)=\int_{0}^{\infty} x^{s-1}\left\{2 \int_{0}^{\infty} \sin x t\left(\frac{1}{e^{2 \pi t}-1}-\frac{1}{2 \pi t}\right) d t\right\} d x
$$

which, with the substitutions $2 \pi t=\tau$ and $x=2 \pi \xi / \tau$, becomes

(7) $\Gamma(s) \cdot \zeta(s)=\frac{(2 \pi)^{s}}{\pi} \int_{0}^{\infty} \frac{\sin \xi}{\xi^{1-s}} d \xi \cdot \int_{0}^{\infty}\left(\frac{1}{e^{\tau}-1}-\frac{1}{\tau}\right) \tau^{-s} d \tau$.

The latter integral has the form of (4), $s$ being replaced by $1-s$, while the former integral is of a well known type. Hence we obtain

$$
\begin{aligned}
\Gamma(s) \cdot \zeta(s) & =\frac{(2 \pi)^{s}}{\pi} \int_{0}^{\infty} \frac{\sin \xi}{\xi^{1-s}} d \xi \cdot \Gamma(1-s) \cdot \zeta(1-s) \\
& =\frac{1}{2} \frac{(2 \pi)^{s}}{\Gamma(1-s) \cdot \cos (\pi s / 2)} \cdot \Gamma(1-s) \zeta(1-s)
\end{aligned}
$$

or

$$
\cos \frac{\pi s}{2} \cdot \Gamma(s) \cdot \zeta(s)=\frac{1}{2}(2 \pi) \cdot \zeta(1-s) .
$$

This is the functional equation, which, by analytical continuation, is valid in the whole $s$-plane.

Returning to (4), we notice that this equality can be written as

$$
\begin{aligned}
\Gamma(s) \zeta(s) & =\int_{0}^{\infty}\left(\sum_{1}^{\infty} e^{-n x}-\int_{0}^{\infty} e^{-\nu x} d \nu\right) x^{s-1} d x \quad(0<\operatorname{Re} s<1) \\
& =\int_{0}^{\infty} \int_{0}^{\infty} e^{-u x} d([u]-u) \cdot x^{s-1} d x .
\end{aligned}
$$

It is evident from (8) that the zeta-function in the critical strip is 
generated by the difference of a sum and an integral. It therefore seems not unlikely that the typical difficulties associated with a study of this function in the critical strip are closely related to this fact.

We now integrate the central Stieltjes integral by parts, as

$$
\begin{aligned}
\int_{0}^{\infty} e^{-u x} d([u]-u) & =\left.e^{-u x}([u]-u)\right|_{0} ^{\infty}+x \int_{0}^{\infty}([u]-u) e^{-u x} d u \\
& =x \int_{0}^{\infty}([u]-u) e^{-u x} d u .
\end{aligned}
$$

Substitution of (8a) in (8) and writing $x=\xi / u$ yields

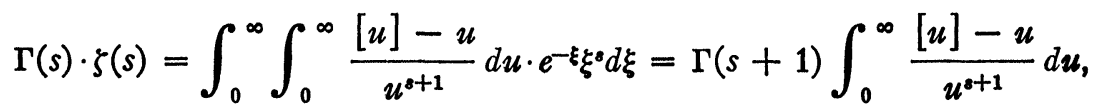

or

$$
\frac{1}{s} \zeta(s)=\int_{0}^{\infty} \frac{[u]-u}{u^{s+1}} d u, \quad 0<\operatorname{Re} s<1 .
$$

Finally, calling $u=e^{x}$ and taking $s=1 / 2+i t$, we obtain from (9) as an expression for $\zeta(s)$ on the critical line $\operatorname{Re} s=1 / 2$ :

$$
\frac{\zeta(1 / 2+i t)}{1 / 2+i t}=\int_{-\infty}^{\infty}\left\{e^{-x / 2}\left[e^{x}\right]-e^{x / 2}\right\} \cdot e^{-i x t} d x .
$$

With (10) the investigation of the zeta-function on the critical line is reduced to a problem in the real domain which can be attacked with physical methods. It is therefore on (10), which has the form of a Fourier transform, that we based our experiments.

The function

$$
y(x)=e^{x / 2}-e^{-x / 2}\left[e^{x}\right]
$$

of which

$$
-\frac{\zeta(1 / 2+i t)}{1 / 2+i t}
$$

is the Fourier transform, is represented in Fig. 1. (See insert opposite p. 980.) It is a sawtooth-function, of which the height of the teeth varies exponentially, the width of the $n$th tooth being given by $\log$ $((n+1) / n)$. Further we have

$$
0 \leqq y(x) \leqq e^{-|x| / 2}, \quad-\infty<x<+\infty,
$$

which ensures the convergence of (10) for all real values of $t$. 
Thus the experimental problem consisted of constructing an electro-mechanical machine giving the Fourier integral of the function $y(x)$. This however was found to be impracticable and therefore we resorted to obtaining experimentally the modulus of the Fourier series for $y(x)$ modified in such a manner that $y(x)$ was taken to extend only from $x_{1}=-9.00$ to $x_{2}=+9.00$, being zero outside these limits. The remaining function was thus repeated indefinitely. It may be noted that the amplitude of $y\left(x_{1}\right)$ and $y\left(x_{2}\right)$ at these limits is only $\exp (-9 / 2)=.011$, so that the error involved is certainly small. The problem thus having been made periodical, it was easy to introduce rotating machinery. For this purpose the function (cut off in this way) was very carefully drawn on paper. This was done radially along the circumference of a circle having a radius of $a=13 \mathrm{~cm}$. (the transformation being $r-a=y(\alpha \zeta))$. Thereupon it was cut out as accurately as possible. The resulting paper disc is represented in Fig. 2 . Thus the first tooth had a width $(\log 2-\log 1)$ corresponding to to about $1 / 26$ of the circumference of the circle. The last tooth which it was practicable to cut out was the 35th with a width of $(\log 36-\log 35)$, corresponding to about $1 / 621$ part of the circumference. This paper disc was clamped perfectly flat between two aluminum discs, the paper teeth extending well outside the metal discs. This assembly was mounted centrally on the shaft of a synchronous motor. The motor was driven at a frequency of 25.018 revolutions per second by an amplifier behind an electrically controlled tuning fork of $1000,72 \mathrm{c} / \mathrm{s}$, the frequency of the current from the tuning fork being demultiplied with the aid of relaxation oscillations in the ratio of $40: 1$. This tuning fork drive ensured a much greater precision in the angular speed of the synchronous motor than could be obtained by using the mains.

With the aid of cylindrical lenses a beam of light was projected onto the paper teeth in a direction parallel to the axis of the motor. The cross section of the beam was a long, very narrow rectangle, with the longer side oriented radially. The light thus passed by the teeth of the disc fell upon a photocell, causing an electric current which was an exact replica of the form of the teeth of the disc and therefore of the modified function. This current was amplified and a strong sinusoidal current was superimposed thereon, the frequency of which was made to vary very slowly in an approximately linear manner from $0 \mathrm{c} / \mathrm{s}$ to $15000 \mathrm{c} / \mathrm{s}$ in about 12 hours, the law of this variation having been previously determined. Further, an effectively quadratic push-pull detector (selenium cells) was inserted in the circuit, producing difference-tones between (a) all the harmonics pro- 
duced by the rotating disc and (b) the applied sinusoidal current. These difference-tones were fed to a very sharply tuned electromechanical vibrator with a natural period of $7.00 \mathrm{c} / \mathrm{s}$ and a logarithmic decrement of .0143 . The variation of the frequency of the applied sinusoidal current was necessarily slow, in order to be sure that the current through the mechanical vibrator went through "quasi-stationary" states. ${ }^{2}$ Thus, when the applied sinusoidal current had a frequency exactly $7.00 \mathrm{c} / \mathrm{s}$ greater or smaller than one of the harmonics generated by the teeth on the disc, a difference-tone was produced which excited the mechanical vibrator. The oscillations of the latter were translated, in a separate circuit, as an electric current which was rectified (giving the modulus of the function) and which was registered by a recording milliammeter. The recording paper of the latter moved with a speed which was also controlled by the same tuning fork. Hence the meter recorded a series of spectral lines situated in pairs, one of each pair being $7 \mathrm{c} / \mathrm{s}$ to the left, and the other $7 \mathrm{c} / \mathrm{s}$ to the right of each harmonic present in the function produced by the rotating disc (fig. 2), which, itself, represents the function $y(x)$ of (11) (made periodical), the modulus of whose Fouriertransform is

$$
\left|\frac{\zeta(1 / 2+i t)}{1 / 2+i t}\right|
$$

Fig. 3 is a direct unretouched reproduction of the record thus obtained of (12). From the accurately known constants of the apparatus it was possible to mark the values of $t$ shown at the top of the record. The abscissae marked at the bottom with a /represent the 29 accurately known zeros of $\zeta(1 / 2+i t)$, which were taken from JahnkeEmde, Funktionen tafeln (zweite, neubearbeite Auflage) p. 324. ${ }^{3}$ It is seen that all these 29 zeros appear in this record either as very deep minima (namely, the zeros no. 1, 2, 3, 4, 5, 6, 7, 11, 12, 13, 16, $18,20,21,23,27,29)$, or in any case as less deep, but none the less very decided minima (namely, the zeros no. 9, 14, 15). It is further seen that the value of the recorded zeros coincide with those calculated with an accuracy better than $1 \%$.

In order that the recording for the larger values should not be too small, the sensitivity of the apparatus was increased 4 -fold from $t=35$

\footnotetext{
${ }^{2}$ Balth. van der Pol, Journal of the Institution of Electrical Engineering (London) vol. 93 (1946) p. 153.

${ }^{3}$ For the number of zeros on $\operatorname{Re} s=1 / 2$ up to $t=1468$ see E. C. Titchmarsh, Proc. Roy. Soc. London Ser. A vol. 151 (1935) p. 234 and vol. 157 (1936) p. 261.
} 




FIG. 1. A graph of the sawtooth function $y(x)=e^{x / 2}-e^{-x / 2}\left[e^{x}\right]$.

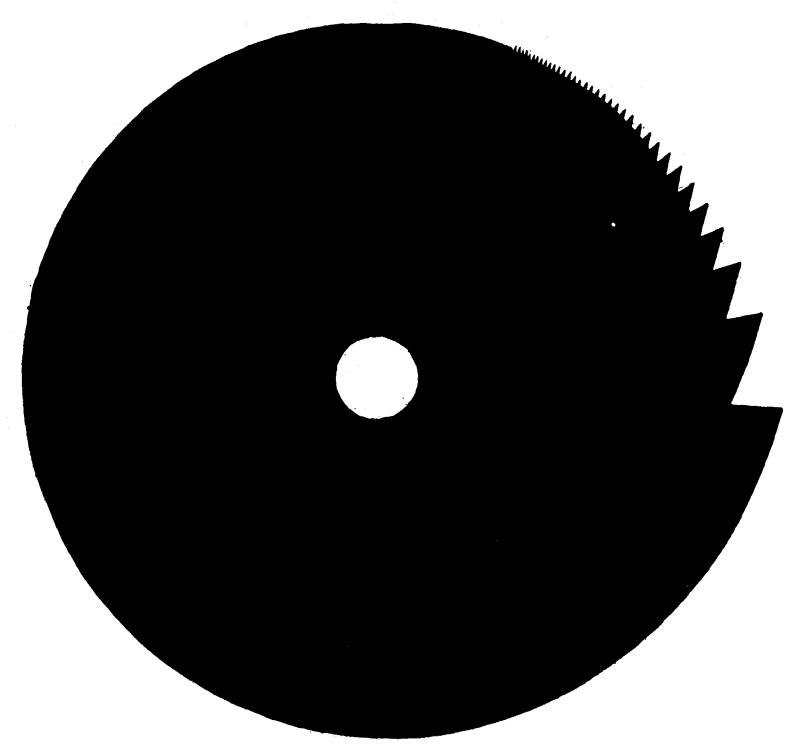

FIG. 2. Paper disc, showing the sawtooth function $y(x)$ for the range $-9<x<+9$. 


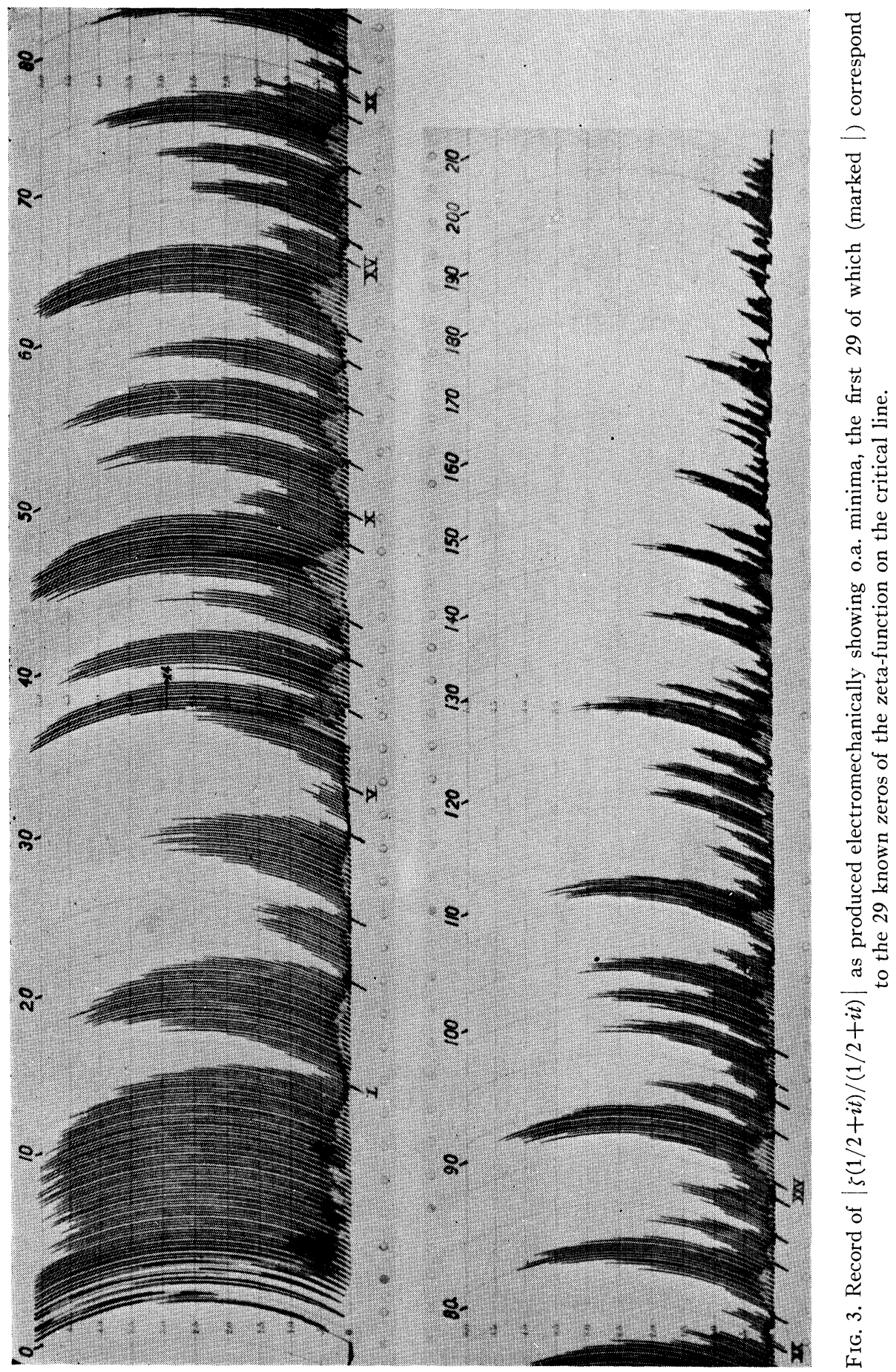


onwards, as indicated in the record, so that for $t>35$, the recorded amplitude is 4 times larger than in the range $t<35$.

The first 29 zeros of $\zeta(1 / 2+i t)$ are to be found in the range $0<t<100$. However it was practically possible to extend the recording up to $t=210$. Up to this point the record shows a total of 73 minima which most probably may be interpreted as zeros, the more so as all the pronounced minima of the envelope of the curve for $t<100$ correspond to known zeros.

As to the modulus, attention may be drawn to the fact that the record does not show $|\zeta(1 / 2+i t)|$, but shows this function divided by $|1 / 2+i t|$, see (12).

It is of interest to remark that our record shows that there seems to be no simple relation between the difference between successive zeros and the height of the maximum between them. For example, the height of the maximum between the 20th and 21 st zero is considerably smaller than that between the 17 th and 18 th, although the interval between these pairs of zeros is not very different. The experiments carried out so far, and in which harmonics beyond the 600th were measured (the highest harmonic recorded corresponded about to the width of the last tooth cut in the paper), were performed with relatively limited means, and it is felt that many improvements could still be applied, such as:

(a) assuring a still more constant speed of revolution of the synchronous motor;

(b) determining the effect on the record of the number of teeth cut in the paper disc, and so on.

Therefore, with the modern technical means available, the present method of exploring the behavior of the zeta-function in the critical strip (which is closely related to Riemann's conjecture), seems capable of improvement with regard to precision. Further, a considerable extension with regard to the number of zeros recorded, including those, if any, outside the line $\operatorname{Re} s=1 / 2$, seems possible by this method.

Finally I wish to thank Mr. C. C. J. Addink for the great care and skill with which he performed the experiments.

Note added in proof (October 6, 1947). The less deep minima as in Fig. 3 were later on found much deeper when in further experiments the angular frequency was kept more homogeneous.

Philips Laboratory, Eindhoven 